\title{
Age-associated impaired plasmacytoid dendritic cell functions lead to decreased CD4 and CD8 $T$ cell immunity
}

\author{
Aishwarya Sridharan • Marc Esposo - Khushboo Kaushal • Jia Tay • \\ Kathyrn Osann • Sudhanshu Agrawal $\cdot$ Sudhir Gupta $\cdot$ Anshu Agrawal
}

Received: 18 August 2010 / Accepted: 27 September 2010 /Published online: 16 October 2010

(C) The Author(s) 2010. This article is published with open access at Springerlink.com

\begin{abstract}
Increased susceptibility to infections, particularly respiratory viral infections, is a hallmark of advancing age. The underlying mechanisms are not well understood, and there is a scarcity of information regarding the contribution of the innate immune system, which is the first line of defense against infections. In the present study, we have investigated the effect of advancing age on plasmacytoid dendritic cell (PDC) function because they are critical in generating a robust antiviral response via the secretion of interferons (IFN). Our results indicate that PDCs from the aged are impaired in their capacity to secrete IFN-I in response to influenza virus and CPG stimulation. Additionally, we observed a severe reduction in the production of IFN-III, which plays an important role in defense against viral infections at respiratory mucosal surfaces. This reduction in IFN-I and IFN-III were a result of ageassociated impaired phosphorylation of transcription factor, IRF-7. Furthermore, aged PDCs were observed
\end{abstract}

A. Sridharan $\cdot$ M. Esposo $\cdot$ K. Kaushal $\cdot$ J. Tay $\cdot$

S. Agrawal $\cdot$ S. Gupta $\cdot$ A. Agrawal $(\square)$

Division of Basic and Clinical Immunology,

Department of Medicine, University of California,

Irvine, CA 92697, USA

e-mail: aagrawal@uci.edu

K. Osann

Department of Medicine, University of California, Irvine, CA 92697, USA to be impaired in their capacity to induce perforin and granzyme in CD8 T cells. Comparison of the antigenpresenting capacity of aged PDC with young PDC revealed that PDCs from aged subjects display reduced capacity to induce proliferation and IFN-gamma secretion in CD4 and CD8 T cells as compared with PDCs from young subjects. In summary, our study demonstrates that advancing age has a profound effect on PDC function at multiple levels and may therefore, be responsible for the increased susceptibility to infections in the elderly.

Keywords Plasmacytoid dendritic cells · Aging · Influenza · Type I interferons · Type III interferons . CD8 cytotoxicity $\cdot \mathrm{T}$ cell proliferation

\section{Introduction}

Aging is characterized by an increased susceptibility to infectious diseases as the ability to fight new infections decreases, and latent infections re-emerge and increase in severity. Infections, such as influenza, pneumonia, and respiratory syncytial virus (RSV) (Powers 1994; Treanor and Falsey 1999; Gavazzi et al. 2004), thus account for nearly one third of deaths in the aged population (Groen et al. 1998; Schildgen 2009). Alterations in the adaptive immune system, particularly the T cells, are thought to account for these age-related 
immune deficiencies. Studies have shown that aged subjects exhibit significantly lower CD8+ cytotoxic responses to influenza virus when compared with young subjects (Mbawuike et al. 1990, 1993; Powers and Belshe 1993; Christensen et al. 2000; Effros et al. 2003; Yang et al. 2005; Jiang et al. 2009). Furthermore, IFN- $\gamma$ secretion from both CD4 and CD8 T cells also decreases with age (Powers and Belshe 1993; Castle et al. 1997; Kang et al. 2004). Although the decline in $\mathrm{T}$ cell immunity is considered a key factor in the increased susceptibility to infections in the elderly, the impact of aging on the innate immune system is not well understood (Pawelec et al. 1998; Solana et al. 2006). Since the innate immune system plays a crucial role in controlling pathogen invasion, and in instructing the adaptive immune system to initiate an antigenspecific response, it is important to determine the extent to which aging affects the innate immune responses.

Plasmacytoid dendritic cells (PDCs) are innate immune cells that are key players in the establishment of an effective immune response against invading pathogens, particularly viruses. PDCs are present in the peripheral blood $(0.2-0.5 \%)$ and secondary lymphoid organs and express the IL-3 receptor (CD123). They are characterized by their plasma cell-like morphology, low phagocytic capacity, and copious production of type I IFNs in response to viral, bacterial, and parasitic infections (Cella et al. 2000; Liu 2005). A single cell is able to produce 1-2 IU of IFN in response to viral stimulus, an amount that is 10-100 times more than most other cells. PDCs owe their ability to detect and respond to microbes to a specific repertoire of Toll-like receptors (TLRs), TLR7 and TLR9 (Takeuchi et al. 2004; Ito et al. 2005; Seeds et al. 2006), which are located in the endosomes and recognize certain nucleotide motifs in viral and bacterial RNA and DNA (Hemmi et al. 2003; Diebold et al. 2004; Heil et al. 2004), respectively. TLR9 recognizes unmethylated 2'-deoxyribo (cytidine-phosphate-guanosine) (CpG) DNA motifs (Hemmi et al. 2000) that are present at high frequencies in pathogens such as viruses and bacteria but are rare in vertebrates. TLR7 recognizes viral single-stranded RNA (ssRNA; influenza) and imidazaquinolones (Hemmi et al. 2002). Unlike other receptors, TLR7 and TLR9 can sense inactivated viruses and thus do not require infection of PDC by viruses to be activated. This is beneficial, as many viruses have developed mechanisms to block the production of type I IFNs early after infection. PDCs constitutively express high levels of the transcription factor IRF-7, which allows rapid production of IFNs in response to infections (Honda et al. 2005). IFNs are considered the first line of defense against pathogens because of their ability to orchestrate numerous biological and cellular processes. Type I IFNs enhance the cytotoxic activity of natural killer and CD8 T cells (Biron 1998; Stetson and Medzhitov 2006; Fitzgerald-Bocarsly and Feng 2007) and regulate the secretion of IFN- $\gamma$ by both CD8 and CD4 T cells (Biron 1998, 2001; O'Shea and Visconti 2000; Stetson and Medzhitov 2006).

Recent studies have reported that PDCs also produce type III IFNs (IL28/29 or IFN $\lambda$ ) in response to viruses (Coccia et al. 2004). Studies of the molecular events leading to type III IFN (IFN-III) production suggest a common mechanism with IFN-I (Ank et al. 2006; Onoguchi et al. 2007). IFN-III signal through a receptor complex composed of a unique IFN- $\lambda \mathrm{R} 1$ chain in addition to a shared IL-10R2 chain, which is also the second subunit of the IL-10, IL-22, and IL-26 receptor complexes (Kotenko et al. 2003). However, whereas IFN-I receptors are expressed in most cell types, IFN$\lambda \mathrm{R} 1$ demonstrates a more restricted pattern of expression, limiting the response of IFN-III to primarily epithelium-like tissues (Witte et al. 2009; Khaitov et al. 2009; Mordstein et al. 2010). Using mice lacking functional receptors for type I IFN, type III IFN, or both, it was observed that IFN-III plays an important role in the defense against several human pathogens that infect the respiratory tract, such as influenza A virus, respiratory syncytial virus, and SARS Coronavirus (Mordstein et al. 2010). Many studies (Shodell and Siegal 2002; Stout-Delgado et al. 2008; Jing et al. 2009; Canaday et al. 2010; Panda et al. 2010) document impairment in the secretion of IFN-I by PDCs from aged subjects in response to viruses. Nevertheless, there is a scarcity of information on the status of IFN-III secretion by PDCs from aged subjects. Age-related alterations in IFN-III secretion may therefore be of great relevance since infection of soft or mucosal tissues are most common in the elderly.

Besides secretion of antimicrobial cytokines, activation of PDCs by TLR7/9 ligands also leads to upregulation of co-stimulatory as well as MHC class I and class II molecules on the PDCs. Upon maturation, PDCs lose their capacity to produce IFNs, acquire a 
myeloid DC-like morphology and initiate adaptive immune responses leading to CD4 and CD8 T cell activation (Gibson et al. 2002). There is no published data regarding the effect of age on the antigenpresenting function of PDCs. Age-associated impairment or reduction in PDC antigen presenting function may also contribute to the increased susceptibility to viral and bacterial infections in the elderly.

In the present study, we report age-associated perturbations in PDC function at three levels: first, at the level of induction of IFN-I and IFN-III via viral and TLR stimulation; secondly, at the level of induction of cytoxicity in CD8 T cell; and finally, at the level of antigen presentation or priming and proliferation of CD4 and CD8 T cells.

\section{Materials and methods}

\section{Blood donors}

Blood was collected from healthy aged (65-90 years) and young (20-35 years) donors. Aged subjects were of middle-class socio-economic status and were living independently. Cohort description is provided in Table 1. The Institutional Review Board of the University of California, Irvine, approved this study.

\section{Isolation and purification of PDCs}

Peripheral Blood Mononuclear Cells were separated from the blood using Ficoll density gradient centrifugation. PDCs were then positively selected using BDCA-4 PDC purification kit from Miltenyi Biotech.

Table 1 Description of the aged and young cohorts

\begin{tabular}{|c|c|c|}
\hline & Young, $n=25$ & Aged, $n=25$ \\
\hline Age (range) & $25(20-35)$ & $80(65-89)$ \\
\hline Gender, female & $15(60 \%)$ & $18(72 \%)$ \\
\hline \multicolumn{3}{|l|}{ Comorbidities } \\
\hline Arthritis & 0 & $14(56 \%)$ \\
\hline Hypertension & 0 & $9(36 \%)$ \\
\hline Dyslipidemia & 0 & $11(44 \%)$ \\
\hline Diabetes & 0 & 0 \\
\hline \multicolumn{3}{|l|}{ Medications } \\
\hline Vitamins & 0 & $19(76 \%)$ \\
\hline Antioxidants & 0 & $14(56 \%)$ \\
\hline
\end{tabular}

This kit contains magnetic beads coupled to BDCA-4, which is expressed exclusively on PDCs. We routinely recovered approximately $0.5-1 \times 10^{5}$ PDCs from $40 \mathrm{ml}$ blood. Purity of the PDC population was $>90 \%$.

Stimulation of PDCs

PDCs $2 \times 10^{4}$ from aged and young subjects were stimulated with either $10 \mu \mathrm{g} / \mathrm{ml}$ heat-inactivated influenza virus A/PR/8/34-INF (Charles River, North Franklin, CT), or with $50 \mu \mathrm{g} / \mathrm{ml} \mathrm{CPG2395}$ (Coley Pharmaceuticals, Wellesley, MA). The RNA from the influenza is a TLR7 ligand, and the synthetic unmethylated oligonucleotide of CPG2395 is a TLR9 ligand. After overnight stimulation at $37^{\circ} \mathrm{C}$, PDCs were centrifuged, and the supernatants were collected and stored at $-70^{\circ} \mathrm{C}$ for cytokine measurement by multiplex cytokine assay (Millipore, San Jose, CA). IFN-III (IL-29) in the supernatants was measured using specific ELISA kit (PBL Biomedicals, Piscataway, NJ).

Flow cytometry staining of PDCs and TLRs

Two hundred microliters of whole blood was incubated with CD303-(BDCA-2) APC, and CD123 PE antibodies for $20 \mathrm{~min}$ at room temperature (RT) to stain for PDCs. RBCs were lysed using BD FACS lysing Solution (BD Biosciences) and fixed with 2\% PFA. The cells were subsequently permeabilized for 30 min at RT using the BD perm buffer. TLR7 and TLR9 antibodies (Imgenex, SanDiego, CA) were added and the stained PDCs were acquired after washing. Flow jo (Treestar Inc.) was used to analyze gated CD303- and CD123-positive cells.

\section{Polymerase chain reaction (PCR)}

Purified PDCs from six aged and six young subjects were pooled, and RNA was extracted using Tri Reagent kit (Molecular Research Center Inc., Cincinnati, OH, USA), following the manufacturer's protocol. Real time PCR was performed with the Mx3005P QPCR System instrument (Stratagene) using the TaqMan Gene expression assay with a FAM ${ }^{\mathrm{TM}}$ dye-labeled TaqMan ${ }^{\circledR}$ MGBprobe and two polymerase chain reaction PCR primers (Applied Biosystems). Briefly, 1 ug of total RNAs were reverse-transcribed using the RT-PCR kit (Applied Biosystems), following the manufacturer's 
instructions. Each sample was amplified in duplicate, and the Glyceraldehyde-3-phosphate dehydrogenase (GAPDH) gene was used for normalization.

Phospho IRF-7 staining

PBMCs from aged and young donors were stimulated with CPG or influenza for $30 \mathrm{~min}$ at $37^{\circ} \mathrm{C}$. Cells were then fixed with $2 \%$ PFA for $10 \mathrm{~min}$ at RT, and subsequently incubated with CD303-(BDCA-2) APC (Miltenyi Biotech) and CD123 PE (BD Biosciences, San Jose, CA) antibodies for $20 \mathrm{~min}$ at RT to stain for PDC surface markers. After washing, PDCs were permeabilized with phospho perm buffer I (BD Biosciences) and stained with phospho IRF-7-
ALEXA 488 (BD Biosciences) for $30 \mathrm{~min}$ at RT. Mouse IgG1-Alexa 488 (BD Biosciences) was used as isotype control. Stained PDCs were acquired on FACsCalibur and gated CD123 plus CD303 doublepositive PDCs were analyzed for phospho IRF-7 using Flow jo.

Perforin and granzyme induction in CD8 T cells

PDCs were purified from PBMCs from aged and young donors, as described in Fig. 1. CD8 T cells from young donors (to rule out any $\mathrm{T}$ cell defect) were purified via magnetic beads using negative selection (Stem Cell Sep). PDCs $1 \times 10^{4}$ were cultured together with $1 \times 10^{5} \mathrm{CD} 8 \mathrm{~T}$ cells in $96-$ well plates, and the
Fig. 1 PDCs from aged subjects are impaired in their capacity to secrete IFN-I and IFN-III. Purified PDCs from aged and young subjects were stimulated with CPG or heat-killed influenza (Flu). Cytokine secretion was determined after overnight stimulation. a Bar diagram depicts the level of type I IFN secretion by aged and young PDCs. b Bar diagram depicts the level of type III IFN secretion by aged and young PDCs. c Bar diagrams depict the levels of TNF- $\alpha$, IL-6, IL-10, and IP-10 secretion by aged and young PDCs. Figure is mean $\pm \mathrm{SE}$ of 25 different aged and young subjects

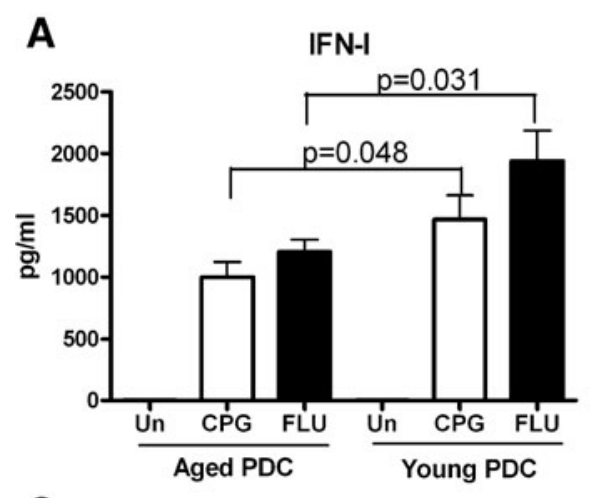

C

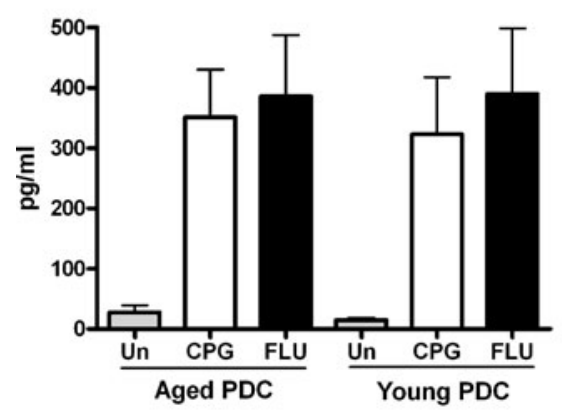

IP-10

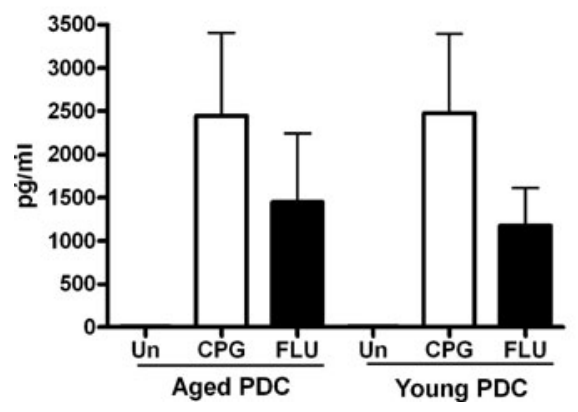

B IFN-III

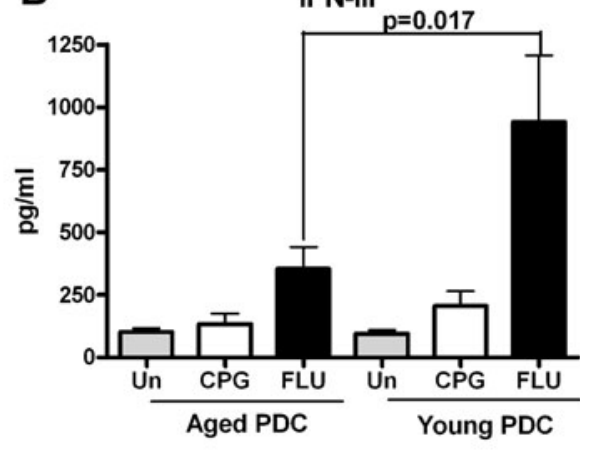

IL-6

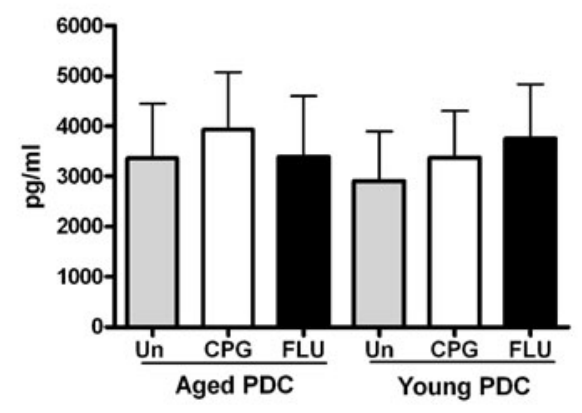

IL-10

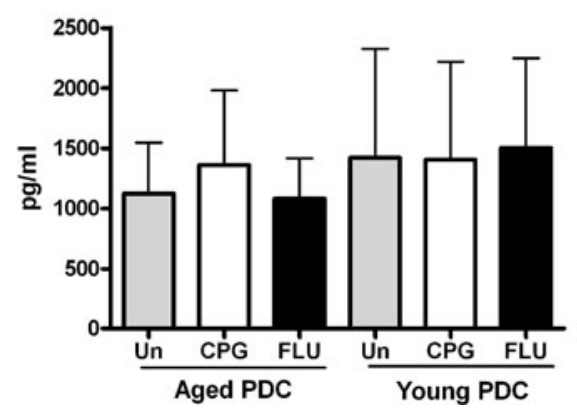


cells were stimulated with CPG or influenza at the described concentrations. Five days later, supernatant was collected, and the cells were surface-stained for CD8. After fixing and permeabilizing, the cells were stained with perforin and granzyme antibodies (BD biosciences), and appropriate isotype controls were used. Flow jo was used to analyze gated CD8 T cells for presence of these granules. Supernatant collected earlier was assayed for IFN- $\gamma$ secretion from CD8 T cells using specific ELISA kit (BD Biosciences).

\section{CD4 and CD8 T cell proliferation}

PDCs were purified from PBMCs of aged and young donors and stimulated with CPG and influenza, as described. After overnight stimulation, supernatants were collected and stored at $-20^{\circ} \mathrm{C}$ for cytokine estimation using the multiplex cytokine assay (Millipore). Stimulated PDCs were cultured with purified, CFSE-labeled allogeneic $T$ cells from young donors at a ratio of 1:20. Six days after culture, cells were collected and stained for CD4 and CD8 markers. Proliferation of gated CD4 T and CD8 T cells was determined by measuring the dilution of CFSE dye. Supernatant collected was assayed for secretion of IFN- $\gamma$ and IL-10 by specific ELISA.

\section{Statistical analysis}

Statistical analysis was performed by a statistician. Within-group differences between unstimulated and stimulated conditions were tested using paired $t$ tests. Mann Whitney or Wilcoxon signed-rank test was used to measure significance between aged and young groups. Values of $p<0.05$ were considered significant.

\section{Results}

PDCs from aged subjects are impaired in their ability to secrete IFN-I and IFN-III (IL-29) following stimulation with CPG and influenza virus

Secretion of IFNs by PDCs is critical in fighting microbial infections. Reports from the literature suggest that PDCs from aged secrete less IFN- $\alpha$ as compared with PDCs from young subjects. Various mechanisms such as reduced numbers of PDCs have been suggested as the culprit. We have previously reported that the percent of PDCs is comparable in the blood of aged and young subjects (Agrawal et al. 2007). To further confirm that reduced IFN secretion is not a consequence of reduction in numbers of PDC with age, in the present study, we have compared IFN secretion from equal numbers of purified aged and young PDCs.

Subjects are described in Table 1. The control population included 25 individuals in the age range of 20-35 years with an average age of 25 years. The geriatric population consisted of 25 individuals in the age range of $65-89$ years with an average age of 80 years. The younger individuals were healthy and not on medications.

As shown in Fig. 1a, secretion of IFN-I in response to $\mathrm{CPG}(\mathrm{Ag} \sim 1,057 \mathrm{pg} / \mathrm{ml}, \mathrm{Yg} \sim 1,584 \mathrm{pg} / \mathrm{ml}, \quad p=$ $0.048)$ and influenza $(\mathrm{Ag} \sim 1163 \mathrm{pg} / \mathrm{ml}, \mathrm{Yg} \sim$ $1941 \mathrm{pg} / \mathrm{ml}, p=0.032$ ) was significantly impaired in PDCs from the elderly when compared with PDCs from the young. Recent reports suggest that secretion of IFN-III or IL-28/29 by PDCs also plays an important role in fighting microbial infections, particularly at mucosal surfaces (Coccia et al. 2004; Mordstein et al. 2010). Since IFN-III induction requires similar signals as IFN-I (Ank et al. 2006; Onoguchi et al. 2007), we determined if there was also an age-associated reduction in IFN-III secretion (Fig. 1b). We observed a highly significant reduction in the levels of IFN-III secretion by aged PDCs in response to influenza $(\mathrm{Ag} \sim 350 \mathrm{pg} / \mathrm{ml}, \mathrm{Yg} \sim 908 \mathrm{pg} / \mathrm{ml}$, $p=0.017$ ) as compared with young subjects. The level of IFN-III reduction in the aged was more pronounced than the age-related decrease observed for IFN-I. In contrast to influenza, CPG induced low levels of IFNIII. Although CPG stimulation showed reduced IFN-III production by aged PDCs compared with young PDCs, the difference was not significant ( $\mathrm{Ag} \sim$ $125 \mathrm{pg} / \mathrm{ml}, \mathrm{Yg} \sim 200 \mathrm{pg} / \mathrm{ml}, p=0.19)$.

A multiplex assay for a panel of other cytokines and chemokines was also performed. IL-6, IL-10, TNF- $\alpha$, and IP-10 were detected after stimulation of PDCs with CPG and influenza. However, there were no significant differences $(p>0.05)$ in the secretion of these cytokines and chemokines between the aged and young PDCs (Fig. 1c). These data demonstrate that PDCs from aged subjects show a selective impairment in their ability to secrete IFN-I and IFN-III in response to influenza virus. Since the impairment of cytokine secretion by aged PDCs is restricted to IFNs it suggests that the uptake of ligands by the TLRs is 
not compromised with age and the reduced IFN secretion may be a consequence of age-associated alterations in the signaling pathway responsible for production of IFN-I and IFN-III.

In the aged subjects, there were several subgroups based on comorbidities. For some of the subgroups, we had enough subjects to do a subgroup analysis. Arthritis was the most common comorbid condition in the aged population studied. IFN-I and IFN-III levels were comparable between arthritis-positive and arthritis-negative subjects $(p=0.78$ IFN-I, $p=0.44$ IFN-III). Aged subjects with hypertension and dyslipidemia were also not different in their induction of IFN-I and IFN-III $(p>0.8)$. Many of the aged subjects were also taking vitamins and antioxidants; however, we did not observe any difference in IFN levels between the two groups $(p>0.9)$. Based on these subgroup analyses, we feel fairly confident that the comparisons between the young control and aged subject populations are yielding valid results across a general geriatric group.

The expression of TLRs is comparable between aged and young subjects

Although our results in Fig. 1 suggested that reduced IFN secretion by aged PDC might not be a consequence of reduced TLR expression, we nevertheless compared the expression of TLR7 and TLR9 in PDCs from aged and young subjects to completely rule out this possibility. As shown in Fig. 2a, b, there was no significant difference $(p>0.05)$ in the expression of either TLR7 or TLR9 in PDCs from aged and young subjects. These data suggest that age-associated alterations in signaling events downstream of TLRs may account for the reduced IFN secretion by PDCs.

PDCs from aged subjects exhibit reduced phosphorylation of IRF-7 in response to CPG and influenza virus

Activation of TLR7 or TLR9 in PDCs leads to MyD88 activation and subsequent recruitment of TRAF6 (TNF receptor associated factor 6), IRAK-4 (interleukin-1 receptor-associated kinase 4), IRAK-1 (interleukin-1 receptor-associated kinase 1), and IRF7 (interferon regulatory factor-7; Kawai et al. 2004; Honda et al. 2004). IRAK-4 phosphorylates IRAK-1, which in turn phosphorylates IRF-7. Once phosphor-
A TLR7

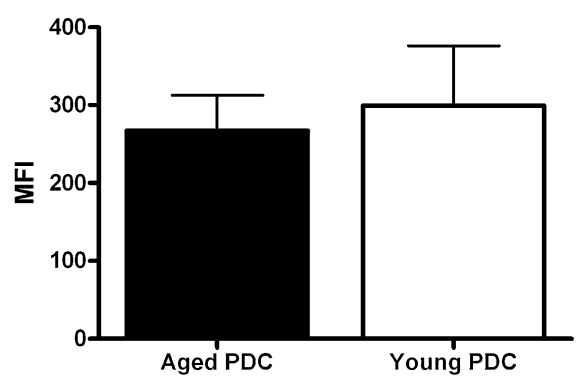

B

TLR9

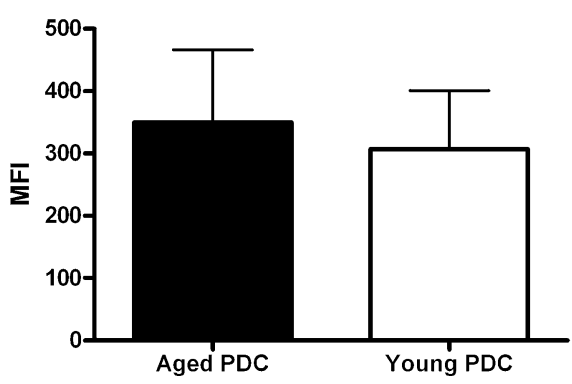

Fig. 2 The expression of TLR7 and TLR9 in PDCs are comparable between aged and young subjects. Intracellular expression of TLRs 7 and 9 was determined by flow cytometry. a Bar diagram depicts the mean fluorescence intensities (MFI) of TLR7 in aged and young PDCs. b Bar diagram depicts the mean fluorescence intensities (MFI) of TLR9 in aged and young PDCs. Figure is mean \pm SE of 12 different aged and young subjects

ylated by IRAK-1, IRF-7 translocates to the nucleus and initiates transcription of genes encoding IFN-I and IFN-III. The formation of the IRF-7, IRAK-1, and MyD88 complex is thus essential for IFN-I and IFN-III production. To investigate whether reduced IFN secretion by aged PDCs is due to a reduced expression of any of these signaling and adaptor molecules, we determined the level of expression of IRF-7, MyD88, and IRAK-1 between aged and young PDCs using real-time (RT) PCR. As shown in Fig. 3a, IRF-7, MyD88, and IRAK-1 gene expression levels are comparable between aged and young subjects, suggesting that the decrease in the expression of these genes is not responsible for the reduced IFN-I and IFN-III secretion in the aged.

It has been observed that type I IFN receptor (IFNAR) deficient PDCs fail to produce type I IFNs in response to TLR agonists, and that IFNAR signaling itself further augments IFN-I production in a positive feedback loop (Watarai et al. 2008). Therefore, we investigated if the expression of IFNAR1, and two 
Fig. 3 Phosphorylation of IRF-7 is impaired in PDCs from aged subjects. a Bar diagram depicts the ratio of IRAK-1, MyD88, IRF-7, IFNAR1, and IFNAR2 to GAPDH in PDCs from aged and young as determined by real-time PCR. b Histograms denote the phosphorylation of IRF-7 at $30 \mathrm{~min}$ in unstimulated and CPG and influenza (Flu)-stimulated aged and young PDCs. Figure is representative of 12 similar experiments. Solid grey histograms depict the isotype; black lined histogram is the experimental group. c Bar graph depicts the mean fluorescence intensity (MFI) minus the isotype MFI of Phospho IRF-7 30 min poststimulation with CPG and influenza. Figure is mean \pm SE of 12 different aged and young subjects
A

RTPCR
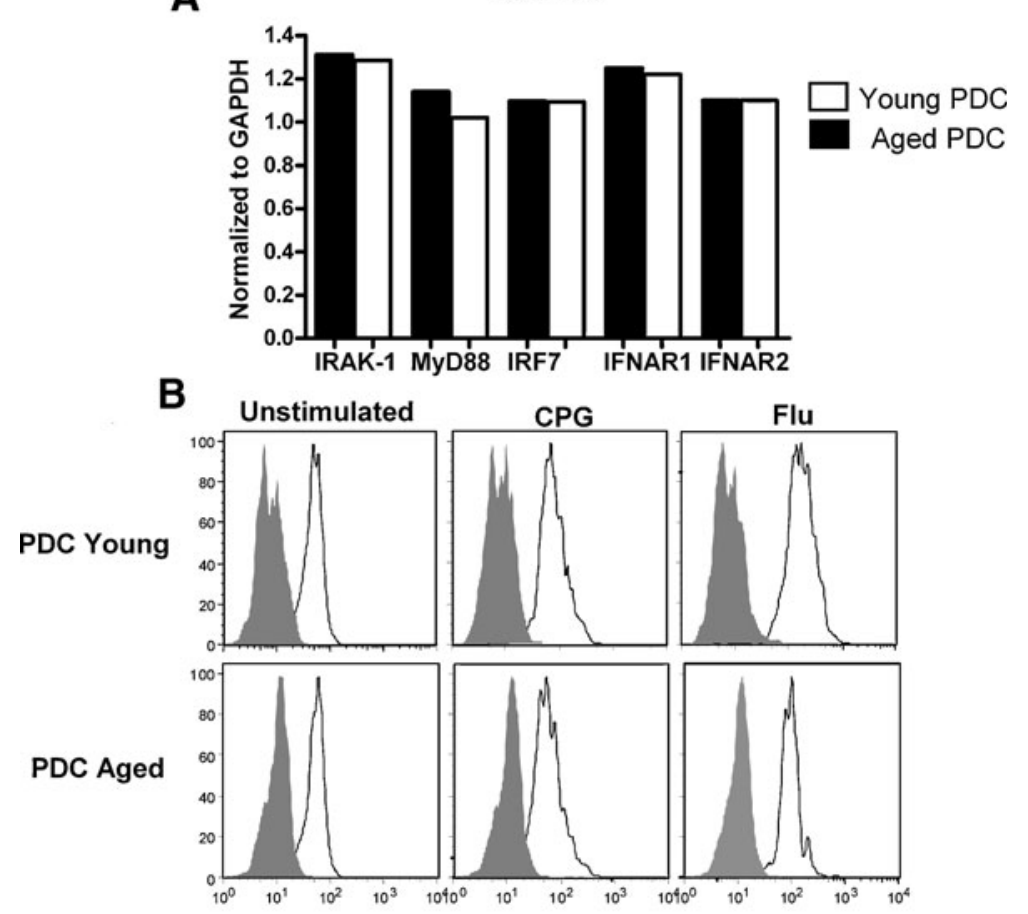

Phospho IRF-7

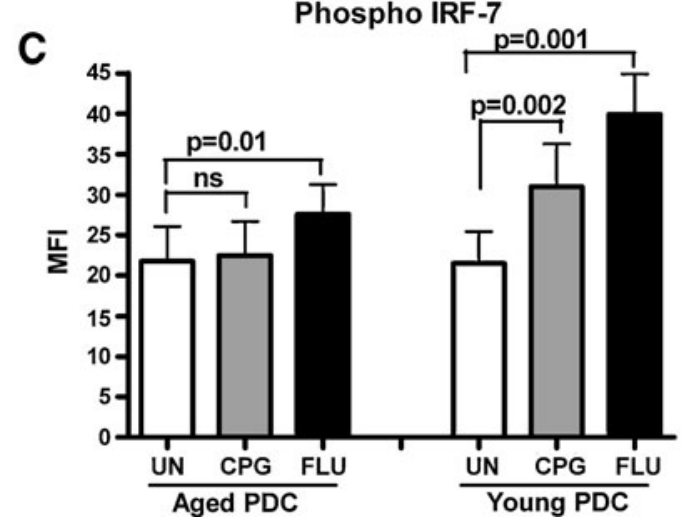

for influenza) IRF-7 phosphorylation compared with unstimulated controls (Fig. 3b, c) in young subjects. In the aged PDCs, the phosphorylation of IRF-7 was severely impaired (Fig. 3b, c). Stimulation with influenza resulted in significantly increased $(p=0.01)$ phosphorylation of IRF-7 in aged PDCs. Stimulation with CPG resulted in an even lower IRF-7 phosphorylation and there was no significant $(p>0.05)$ upregulation compared with unstimulated controls. These results clearly demonstrate that the PDCs from the aged subjects are impaired in their ability to phosphorylate IRF-7, which may be responsible for the reduced IFN-I and IFN-III production. 
PDCs from aged subjects are impaired in their capacity to induce perforin, granzyme and IFN- $\gamma$ producing CD8 $\mathrm{T}$ cells

Activation of PDCs by viruses is considered a key event in enhancing the cytotoxicity of CD8 T cells by inducing cytotoxic granules of perforin and granzyme, predominantly through IFN-I and IFN-III secretion (Tiwari et al. 1991; Kolumam et al. 2005). Since IFN-I and IFN-III production is decreased in aged PDCs, we investigated if the decrease was sufficient to impair the generation of cytotoxic CD8 $\mathrm{T}$ cells. As shown in Fig. 4a, c (contour plots), influenza-stimulated young and aged PDCs resulted in an increased percentage of CD8 T cells expressing perforin and granzyme relative to the unstimulated PDCs. However, the percent of CD8 T cells expressing perforin and granzyme over unstimulated controls was significantly less ( $p=0.035$ for perforin, $p=0.005$ for granzyme) in aged PDCs as compared with that in young PDCs (Fig. 4b, d). Stimulation of aged PDCs with CPG did not result in a significant increase $(p>$ 0.05) in the percentage of CD8 T cells expressing perforin and granzyme over unstimulated controls. However, CPG stimulation of young PDCs showed a significant increase $(p<0.05$, Fig. $4 \mathrm{a}, \mathrm{c})$ in the percentage of perforin and granzyme expressing CD8 T cells. Therefore, percent of CD8 T cells expressing perforin and granzyme over unstimulated controls was significantly less ( $p=0.049$ for perforin, $p=0.015$ for granzyme) in aged PDCs as compared with that in young PDCs (Fig. 4b, d).

In addition to perforin and granzyme, IFN- $\gamma$ production by $\mathrm{CD} 8 \mathrm{~T}$ cells is also essential for mounting a robust antiviral response. Therefore, we used ELISA to assay the IFN $-\gamma$ released by the CD8 $\mathrm{T}$ cells after exposure to the stimulated young and aged PDCs. IFN- $\gamma$ secretion displayed a profile very similar to perforin and granzyme (Fig. 4e, f). Figure $4 \mathrm{e}$ depicts the level of IFN- $\gamma$ secreted by CD8 T cells while Fig. 4f represents the fold increase which is derived by calculating the increase in IFN- $\gamma$ secretion on stimulation over the unstimulated values. IFN- $\gamma$ secretion was significantly reduced $(p=0.01)$ in CD8 T cells co-cultured with influenza-stimulated aged PDCs relative to influenza-stimulated young PDCs. IFN $-\gamma$ secretion in response to CPG was also significantly impaired $(p=0.026)$ in the aged subjects relative to the young subjects. These data demonstrate that PDCs from aged are impaired in their capacity to enhance the expansion of cytotoxic CD8 T cells expressing perforin, granzyme, and IFN- $\gamma$.

PDCs from aged subjects are impaired in their capacity to prime CD4 and CD8 T cells

In addition to secretion of IFNs, activation of PDCs by microbial ligands also leads to antigen presentation (Gibson et al. 2002). After initial secretion of IFNs, PDCs subsequently mature, and lose their capacity to produce IFNs and acquire antigen presenting functions. Mature PDCs are capable of priming adaptive immune CD4 and CD8 T cell responses. We investigated the capacity of aged and young PDCs to induce CD4 $\mathrm{T}$ and CD8 $\mathrm{T}$ cell proliferation. Purified aged and young PDCs were stimulated overnight with CPG or influenza virus as described. Next day, the cells were collected and cultured with purified $\mathrm{T}$ cells to determine $\mathrm{T}$ cell proliferation, and cytokine secretion. Although influenza-stimulated aged PDCs induced CD4 and CD8 $\mathrm{T}$ cell proliferation, the proliferation was significantly $(p=0.027$ for CD4 T, $p=0.032$ for CD8 T) reduced when compared with young subjects (Fig. 5d). CPG-stimulated aged PDCs were unable to induce proliferation of $\mathrm{CD} 4 \mathrm{~T}$ cells above the unstimulated controls. Induction of $\mathrm{CD} 8 \mathrm{~T}$ cell proliferation by CPG-stimulated aged PDCs was slightly enhanced over controls though the difference was not significant $(p>0.05)$. In contrast, CPGstimulated PDCs from young donors significantly $(p<0.05)$ enhanced the proliferation of CD4 T cells and CD8 T cells. The CD4 and CD8 T cell proliferation induced by aged and young PDCs was comparable in the absence of stimulation.

Next, we determined the nature of cytokines secreted by $\mathrm{T}$ cells primed with stimulated and unstimulated aged and young PDCs. T cells stimulated with both CPG and flu stimulated aged PDCs displayed higher levels of IL-10 and lower levels of IFN- $\gamma$ compared with T cells primed with their young counterparts though the differences are not significant $(p>0.05)$. IL-5 levels were undetectable (data not shown).

These data suggest that PDCs from aged subjects display reduced capacity to prime $\mathrm{T}$ cell proliferation and cytokine secretion and are therefore defective in antigen presentation. 

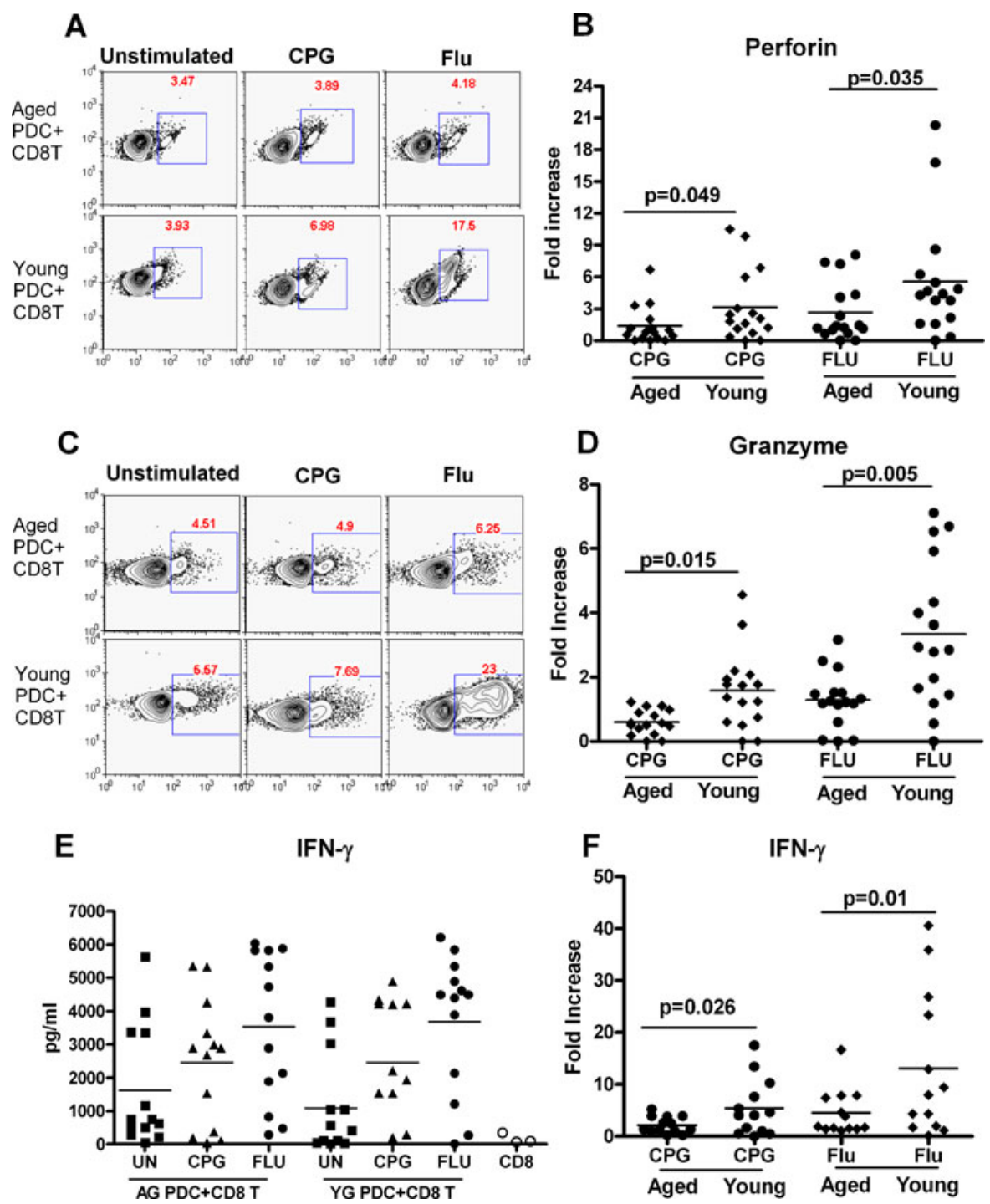

Fig. 4 PDCs from aged are impaired in their capacity to prime cytotoxic CD8 T lymphocyte responses. a Contour plot depicts the intracellular expression of perforin in CD8 T cells cocultured with aged and young PDCs stimulated with CPG and influenza (Flu). Figure is representative of 15 such experiments. b Graph depicts the fold induction of perforin in CD8 T cells cultured with CPG or influenza-stimulated aged and young $\mathrm{PDCs}$ compared with unstimulated PDCs. Figure is mean $\pm \mathrm{SE}$ of 15 different aged and young subjects. Each dot corresponds to one separate subject. c Contour plot depicts the intracellular expression of granzyme in CD8 T cells co-cultured with aged and young PDCs stimulated with CPG and influenza (Flu). Figure is representative of 15 such experiments. d Graph

\section{Discussion}

Immunosenescence compromises protection against pathogens and contributes to mortality and morbidity depicts the fold induction of granzyme in CD8 T cells cultured with CPG or influenza stimulated aged and young PDCs compared with unstimulated PDCs. Figure is mean \pm SE of 15 different aged and young subjects. Each dot corresponds to one separate subject. e Graph depicts the levels of IFN- $\gamma$ in the supernatant of aged and young PDC-CD8 coculture on stimulation with CPG and influenza (Flu). f Graph depicts the fold induction of IFN- $\gamma$ in CD8 T cells cultured with CPG or influenza-stimulated aged and young PDCs compared with unstimulated PDCs. Figure is mean $\pm \mathrm{SE}$ of 15 different aged and young subjects. Each dot corresponds to one separate subject

in the elderly. The scarcity of information about the mechanisms involved in age-associated immune dysfunction makes it difficult to develop effective treatments to boost innate immunity in the elderly. In 
Fig. 5 PDCs from aged are impaired in their capacity to prime $\mathrm{CD} 4$ and $\mathrm{CD} 8 \mathrm{~T}$ cell responses. a Bar graph depicts the percentage of CD4 $\mathrm{T}$ cells proliferating after culture with CPG and influenza stimulated aged and young PDCs. b Bar graph depicts the fold induction of proliferation in CD4 T cells cultured with CPG or influenza-stimulated aged and young PDCs compared with unstimulated PDCs. c Bar graph depicts the percentage of CD8 T cells proliferating after culture with CPG and influenza-stimulated aged and young PDCs. d $B a r$ graph depicts the fold induction of proliferation in CD8 T cells cultured with CPG or influenza stimulated aged and young PDCs compared with unstimulated PDCs. e Bar graph depicts the level of IFN- $\gamma$ secreted by $\mathrm{T}$ cells. f Bar graph depicts the level of IL-10 secreted by $\mathrm{T}$ cells Figure is mean \pm SE of 12 different aged and young subjects
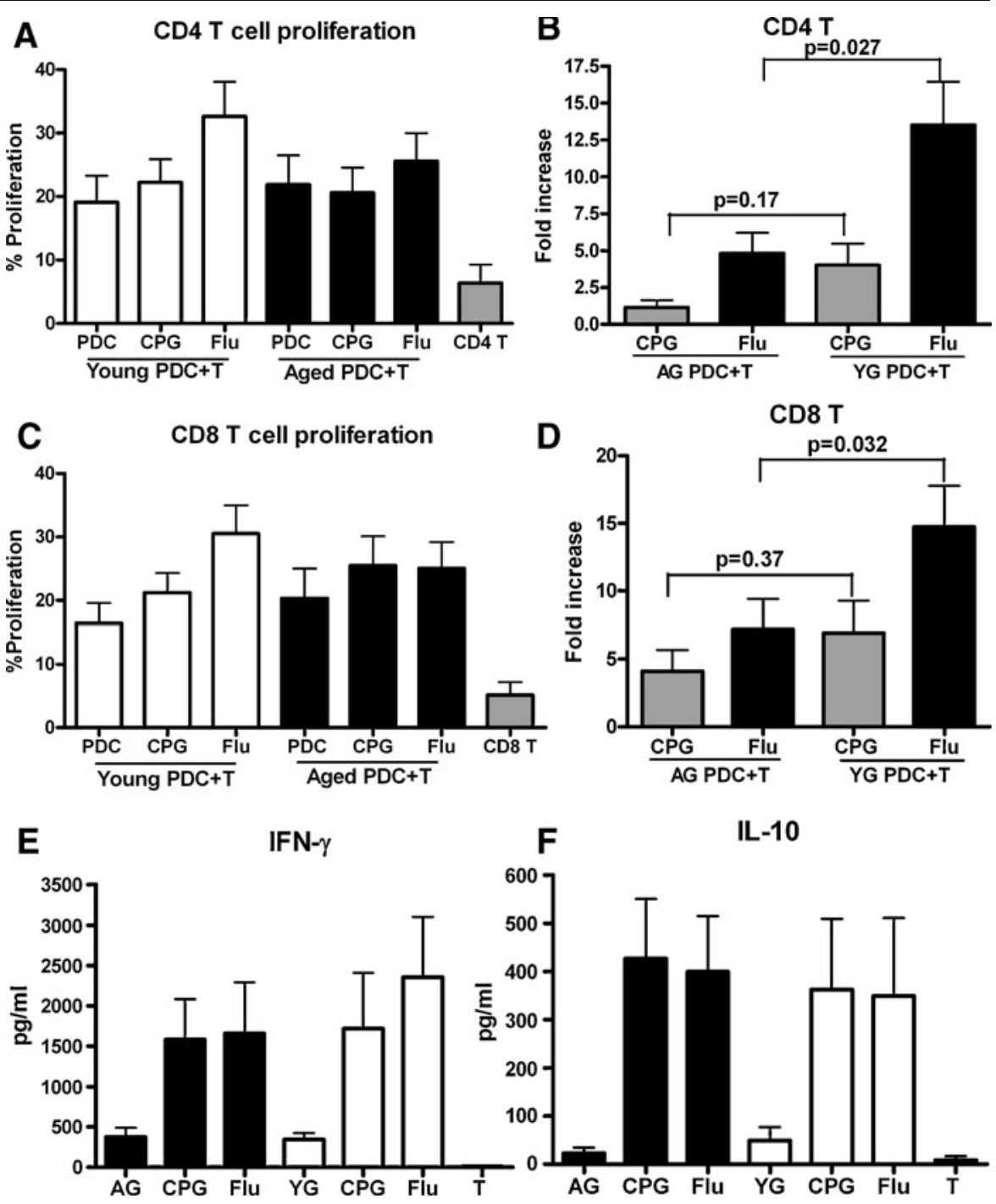

this study, we demonstrate that PDC function declines with age, and the effect of this decline results in reduced $\mathrm{T}$ lymphocyte responses associated with aging.

Aged subjects display increased susceptibility to viral infections particularly of the respiratory mucosa (Powers 1994; Groen et al. 1998; Treanor and Falsey 1999; Gavazzi et al. 2004; Schildgen 2009). PDCs are critical in mounting an effective immune response against viruses because of their ability to secrete very high levels of IFNs (Liu 2005). Here, we demonstrate that purified PDCs from aged subjects secrete reduced quantities of IFN-I after stimulation with $\mathrm{CPG}$ and influenza virus relative to their young counterparts. This finding is consistent with numerous other studies that reported a decline in IFN-I production by PDCs from aged subjects in response to CPG and influenza in humans and mice (Shodell and Siegal 2002; StoutDelgado et al. 2008; Jing et al. 2009; Canaday et al. 2010; Panda et al. 2010). Furthermore, we report a more pronounced decline in the production of IFN-III by aged PDCs in response to influenza. IFN-III is a recently discovered antiviral cytokine that has a similar activity as IFN-I except that its action is restricted to renal and mucosal epithelial surfaces (Dumoutier et al. 2004; Ank et al. 2006; Onoguchi et al. 2007; Sommereyns et al. 2008; Khaitov et al. 2009; Li et al. 2009; Mordstein et al. 2010). The IFNIII system is believed to have been evolved as a specific protection of epithelia and to prevent viral invasion through skin and mucosal surfaces. In a model for a localized Herpes Simplex Virus 2 (HSV-2) 
infection, it was observed that IFN-III completely blocked viral replication in the vaginal mucosa and prevented development of disease. This is in contrast to IFN-I, which had a more modest antiviral activity (Ank et al. 2006). IFN-III displayed moderate antiviral activity against systemic HSV-2 infection emphasizing its role in protection from microorganisms at mucosal surfaces. More recently, Mordstein et al. (Mordstein et al. 2010) observed that IFN-I and IFN-III receptor double-knockout mice showed enhanced susceptibility to various viruses that primarily replicate in lung epithelial cells such as influenza, RSV and SARSCoronavirus. In contrast, they observed that IFN-III did not provide protection against intra-nasal infection with lassa virus. It was suggested this was because the expression of functional IFN-III receptors in the lung was restricted to epithelial cells. Our observation of a severe age-associated decline in the production of IFNIII may explain the increased risk of influenza and other respiratory infections in the elderly. It may also be important in defense against other infections such as those of skin and urinary tract that are prevalent in the elderly. The role of IFN-III has not been previously explored in aging. In contrast to influenza, we did not observe a significant age-associated reduction in IFNIII in response to CPG. This could be due to low levels of induction of IFN-IIIs by CPG. Reports indicate that IFN-III is induced after stimulation with several ssRNA viruses, whereas the information on viruses with other genomes (DNA and double-stranded RNA [dsRNA]) and bacteria is sparse (Brand et al. 2005; Ank et al. 2006).

We consistently found CPG to be much less stimulatory than flu in all our experiments. The response in aged PDCs was even lower than what was observed with young PDCs. It may be one of the factors responsible for the increased susceptibility of the aged to bacterial infections such as pneumonia. However, this finding needs to be confirmed further using whole bacteria since it is possible that purified oligo-dinucleotides such as CPG are not taken up by PDCs as efficiently as a whole virus or bacteria.

Various mechanisms, such as decreased numbers of PDC (Shodell and Siegal 2002; Jing et al. 2009; Canaday et al. 2010), and decreased expression of TLR7 and TLR9 (Jing et al. 2009; Panda et al. 2010), have been suggested for the observed decrease in type I IFN production by aged humans. Contrary to these reports, our observations suggest that decreased IFN-I and IFN-III secretion by aged PDCs is due to an impaired phosphorylation of IRF-7, a key transcription factor responsible for the production of IFNs (Honda et al. 2004). Since PDCs constitutively express high levels of IRF-7 (Fitzgerald-Bocarsly and Feng 2007), activation via TLRs causes the phosphorylation of IRF-7 by IRAK-1. The phosphorylated IRF-7 translocates to nucleus to induce transcription of IFN-I. IFN-III induction in PDCs has also been observed to utilize a similar mechanism (Ank et al. 2006; Onoguchi et al. 2007). Ageassociated declines in the activation of IRF-7 have been shown to be responsible for the decreased IFN- $\alpha$ (IFN-I) secretion in PDCs in mice (Stout-Delgado et al. 2008). Further evidence that reduced expression of the TLR7 and 9 is not responsible for the decline in IFN production with age comes from the fact that aged PDCs display normal secretion of inflammatory cytokines (IL-6, IL-10, TNF- $\alpha$ ) and chemokines (IP10) after TLR stimulation, suggesting that the agerelated defects in cytokine production may be limited to defective IFN signaling pathways.

In addition to reduced IFN secretion, we also observed a severe decline in the induction of perforin, granzyme, and IFN- $\gamma$ production in $\mathrm{CD} 8+\mathrm{T}$ cells cultured with influenza and CPG-stimulated aged PDCs relative to young PDCs. Reduced IFN-I secretion by aged PDCs may account for this decrease. Several studies have demonstrated that IFN-I plays an essential role in the differentiation and function of effector CD8 $\mathrm{T}$ lymphocytes. Kolumam et al. (2005) found that CD8 T cells lacking the type I IFN receptor were profoundly impaired in their ability to expand and differentiate into effector CTLs after LCMV infection, demonstrating that type I IFNs provide a non-redundant costimulatory signal in vivo. Similar to IFN-I, IFNIII can be induced by viral infections and has been observed to have potent antiviral activity against viral infections in vivo (Bartlett et al. 2005; Ank et al. 2006, 2008; Almeida et al. 2008). Several reports have now demonstrated that IFN-III has the ability to inhibit the replication of a number of viruses, including hepatitis $C$ virus (Robek et al. 2005). IFNIII has also been shown to provide protection in mice from genital herpes infection and disease progression (Ank et al. 2006, 2008; Iversen et al. 2010). Type III IFNs are capable of inducing the expression by cells infected with virus of many antiviral proteins, such as 
dsRNA-activated serine/threonine protein kinase, 2',5'-OAS, and MxA (also known as MX1) proteins, which mediate antiviral protection (Brand et al. 2005; Ank et al. 2006). However, whether IFN-III has a direct effect on CD8 cytotoxicity is not clear at present.

PDC are able to differentiate into mature dendritic cells, upon viral stimulation and stimulate allogeneic naïve CD4 T lymphocytes (Cella et al. 2000; Kawamura et al. 2006). Recently, it was shown that not only viruses, but also whole live bacteria can induce PDC activation, resulting in Th1 polarization of naive CD4 T cells (Guiducci et al. 2006). Reduced function of $\mathrm{T}$ cells such as decreased proliferation and decline in IFN- $\gamma$ production are believed to be major mechanisms of increased susceptibility to viral infections in the elderly. The efficiency of PDC as antigenpresenting cells has not been characterized in aging. Our data demonstrate that the PDC's ability to present antigens is severely compromised with age. Aged PDCs are impaired in their ability to induce CD4 and CD8 T cell proliferation and IFN- $\gamma$ secretion (Fig. 5). This suggests that aberrant PDC functions can adversely affect the adaptive immune response in older individuals.

In summary, we demonstrate that advancing age significantly affects the functions of PDCs at all levels from secretion of antiviral cytokines to priming of $\mathrm{T}$ cell responses. Therefore, the age-associated decline in PDC function may be a major factor responsible for the increased susceptibility of elderly individuals to infections. Delineating this functional decline may help in designing optimal therapies to treat infections in the elderly. For example, our findings suggest that administration of type III IFNs may be a novel approach to treating of respiratory viral infections in adults, particularly because the restricted expression of the IFN-III receptor will result in fewer side effects.

Acknowledgements This study is supported by New Scholar grant from the Ellison Medical Foundation. We thank General Clinical Research Center (GCRC), UCI, for providing blood from young donors.

Author contributions AS performed experiments for Figs. 1, 4 , and 5 and contributed to manuscript preparation; ME did flow cytometry staining for IRF-7, KK stained for TLRs; JT did the RTPCR experiments; KO performed statistical analysis; SA contributed to T cell assays; SG provided intellectual support; and AA supervised the work, designed the experiments, analyzed the data, and wrote the manuscript.
Open Access This article is distributed under the terms of the Creative Commons Attribution Noncommercial License which permits any noncommercial use, distribution, and reproduction in any medium, provided the original author(s) and source are credited.

\section{References}

Agrawal A, Agrawal S, Cao JN, Su H, Osann K, and Gupta S (2007) Altered innate immune functioning of dendritic cells in elderly humans: a role of the phosphoinositide 3-kinase-signaling pathway. J Immunol 178(11):69126922

Almeida GM, de Oliveira DB, Magalhaes CL, Bonjardim CA, Ferreira PC, Kroon EG (2008) Antiviral activity of type I interferons and interleukins 29 and 28a (type III interferons) against Apeu virus. Antiviral Res 80(3):302-308. doi:10.1016/j.antiviral.2008.06.016

Ank N, West H, Bartholdy C, Eriksson K, Thomsen AR, Paludan SR (2006) Lambda interferon (IFN-lambda), a type III IFN, is induced by viruses and IFNs and displays potent antiviral activity against select virus infections in vivo. J Virol 80 (9):4501-4509. doi:10.1128/JVI.80.9.4501-4509.2006

Ank N, Iversen MB, Bartholdy C, Staeheli P, Hartmann R, Jensen UB, Dagnaes-Hansen F, Thomsen AR, Chen Z, Haugen H, Klucher K, Paludan SR (2008) An important role for type III interferon (IFN- $\lambda /$ IL-28) in TLR-induced antiviral activity. J Immunol 180(4):2474-2485

Bartlett NW, Buttigieg K, Kotenko SV, Smith GL (2005) Murine interferon $\lambda \mathrm{s}$ (type III interferons) exhibit potent antiviral activity in vivo in a poxvirus infection model. J Gen Virol 86(Pt 6):1589-1596. doi:10.1099/ vir.0.80904-0

Biron CA (1998) Role of early cytokines, including alpha and beta interferons (IFN-alpha/beta), in innate and adaptive immune responses to viral infections. Semin Immunol 10 (5):383-390. doi:10.1006/smim.1998.0138

Biron CA (2001) Interferons alpha and beta as immune regulators-a new look. Immunity 14(6):661-664. doi:10.1016/S1074-7613(01)00154-6

Brand S, Beigel F, Olszak K, Zitzmann S, Eichhorst ST, Otte JM, Goeke B, Diepolder H, Adler B, Auernhammer C, Dambacher J (2005) The novel lambda-interferons IL-28A and IL-29 mediate proinflammatory, antiproliferative, and antiviral signals in intestinal epithelial cells. Gastroenterology 129(1):371. doi:10.1053/j.gastro.2005.05.062

Canaday DH, Amponsah NA, Jones L, Tisch DJ, Hornick TR, Ramachandra L (2010) Influenza-induced production of interferon-alpha is defective in geriatric individuals. J Clin Immunol 30(3):373-383. doi:10.1007/s10875-010-9374-9

Castle S, Uyemura K, Wong W, Modlin R, Effros R (1997) Evidence of enhanced type 2 immune response and impaired upregulation of a type 1 response in frail elderly nursing home residents. Mech Ageing Dev 94(1-3):7-16. doi:10.1016/S0047-6374(96)01821-0

Cella M, Facchetti F, Lanzavecchia A, Colonna M (2000) Plasmacytoid dendritic cells activated by influenza virus and CD40L drive a potent TH1 polarization. Nat Immunol 1(4):305-310. doi:10.1038/79747 
Christensen JP, Doherty PC, Branum KC, Riberdy JM (2000) Profound protection against respiratory challenge with a lethal H7N7 influenza A virus by increasing the magnitude of CD8(+) T-cell memory. J Virol 74(24):1690-1696

Coccia EM, Severa M, Giacomini E, Monneron D, Remoli ME, Julkunen I, Cella M, Lande R, Uzé G (2004) Viral infection and Toll-like receptor agonists induce a differential expression of type I and lambda interferons in human plasmacytoid and monocyte-derived dendritic cells. Eur J Immunol 34 (3):796-805. doi:10.1002/eji.200324610

Diebold SS, Kaisho T, Hemmi H, Akira S, Reis e Sousa C (2004) Innate antiviral responses by means of TLR7mediated recognition of single-stranded RNA. Science 303 (5663):1529-1531. doi:10.1126/science.1093616

Dumoutier L, Tounsi A, Michiels T, Sommereyens C, Kotenko SV, Renauld JC (2004) Role of the interleukin (IL)-28 receptor tyrosine residues for antiviral and antiproliferative activity of IL-29/interferon- $\lambda 1$ : similarities with type I interferon signaling. J Biol Chem 279:32269-32274. doi:10.1074/jbc.M404789200

Effros RB, Cai Z, Linton PJ (2003) CD8 T cells and aging. Crit Rev Immunol 23(1-2):45-64. doi:10.1615/CritRevImmunol.v23. i12.30

Fitzgerald-Bocarsly P, Feng D (2007) The role of type I interferon production by dendritic cells in host defense. Biochimie 89 (6-7):843-855. doi:10.1016/j.biochi.2007.04.018

Gavazzi G, Herrmann F, Krause KH (2004) Aging and infectious diseases in the developing world. Clin Infect Dis 39(1):83-91. doi:10.1086/421559

Gibson SJ, Lindh JM, Riter TR, Gleason RM, Rogers LM, Fuller AE, Oesterich JL, Gorden KB, Qiu X, McKane SW, Noelle RJ, Miller RL, Kedl RM, Fitzgerald-Bocarsly P, Tomai MA, Vasilakos JP (2002) Plasmacytoid dendritic cells produce cytokines and mature in response to the TLR7 agonists, imiquimod and resiquimod. Cell Immunol 218(1-2):74-86. doi:10.1016/S0008-8749(02)00517-8

Groen J, Claas EC, Balentien E, Braakman D, Osterhaus AD (1998) High influenza morbidity and mortality in unvaccinated elderly people in Curacao. J Infect 36 (2):241-242

Guiducci C, Ott G, Chan JH, Damon E, Calacsan C et al (2006) Properties regulating the nature of the plasmacytoid dendritic cell response to Toll-like receptor 9 activation. J Exp Med 203(8):999-2008. doi:10.1084/ jem.20060401

Heil F, Hemmi H, Hochrein H, Ampenberger F, Kirschning C, Akira S, Lipford G, Wagner H, Bauer S (2004) Speciesspecific recognition of single-stranded RNA via toll-like receptor 7 and 8 . Science 303(5663):1526-1529. doi:10.1126/science. 1093620

Hemmi H, Takeuchi O, Kawai T, Kaisho T, Sato S, Sanjo H, Hoshino K, Wagner H, Takeda K, Akira S (2000) A Tolllike receptor recognizes bacterial DNA. Nature 408 (6813):740-745. doi:10.1038/35047123

Hemmi H, Kaisho T, Takeuchi O, Sato S, Sanjo H, Hoshino K, Horiuchi T, Tomizawa H, Takeda K, Akira S (2002) Small anti-viral compounds activate immune cells via the TLR7 MyD88-dependent signaling pathway. Nat Immunol 3 (6):196-200. doi:10.1038/ni758

Hemmi H, Kaisho T, Takeda K, Akira S (2003) The roles of Tolllike receptor 9, MyD88, and DNA-dependent protein kinase catalytic subunit in the effects of two distinct CpG DNAs on dendritic cell subsets. J Immunol 170(6):3059-3064

Honda K, Yanai H, Mizutani T, Negishi H, Shimada N, Suzuki N, Ohba Y, Takaoka A, Yeh WC, Taniguchi T (2004) Role of a transductional-transcriptional processor complex involving MyD88 and IRF-7 in Toll-like receptor signaling. Proc Natl Acad Sci U S A 101(43):15416-15421. doi:10.1073/pnas.0406933101

Honda K, Yanai H, Negishi H, Asagiri M, Sato M, Mizutani T, Shimada N, Ohba Y, Takaoka A, Yoshida N, Taniguchi T (2005) IRF-7 is the master regulator of type-I interferondependent immune responses. Nature 434(7034):772-777. doi:10.1038/nature03464

Ito T, Wang YH, Liu YJ (2005) Plasmacytoid dendritic cell precursors/type I interferon-producing cells sense viral infection by Toll-like receptor (TLR) 7 and TLR9. Springer Semin Immunopathol 26(3):221-229. doi:10.1007/s00281004-0180-4

Iversen MB, Ank N, Melchjorsen J, Paludan SR (2010) Expression of type III interferon (IFN) in the vaginal mucosa is mediated primarily by dendritic cells and displays stronger dependence on NF-kappaB than type I IFNs. J Virol 84 (9):4579-4586. doi:10.1128/JVI.02591-09

Jiang J, Bennett AJ, Fisher E, Williams-Bey Y, Shen H, Murasko DM (2009) Limited expansion of virus-specific CD8 T cells in the aged environment. Mech Ageing Dev 130(11-12):713-721. doi:10.1016/j.mad.2009.08.007

Jing Y, Shaheen E, Drake RR, Chen N, Gravenstein S, Deng Y (2009) Aging is associated with a numerical and functional decline in plasmacytoid dendritic cells, whereas myeloid dendritic cells are relatively unaltered in human peripheral blood. Hum Immunol 70(10):777-784. doi:10.1016/j. humimm.2009.07.005

Kang I, Hong MS, Nolasco H, Park SH, Dan JM, Choi JY, Craft J (2004) Age-associated change in the frequency of memory CD4+ T cells impairs long term CD4+ T cell responses to influenza vaccine. J Immunol 173(1):673-681

Kawai T, Sato S, Ishii KJ, Coban C, Hemmi H, Yamamoto M, Terai K, Matsuda M, Inoue J, Uematsu S, Takeuchi O, Akira S (2004) Interferon-alpha induction through Tolllike receptors involves a direct interaction of IRF7 with MyD88 and TRAF6. Nat Immunol 5(10):1061-1068. doi:10.1038/ni1118

Kawamura K, Kadowaki N, Kitawaki T, Uchiyama T (2006) Virus-stimulated plasmacytoid dendritic cells induce CD4 + cytotoxic regulatory T cells. Blood 107(3):1031-1038. doi:10.1182/blood-2005-04-1737

Khaitov MR, Laza-Stanca V, Edwards MR, Walton RP, Rohde G, Contoli M, Papi A, Stanciu LA, Kotenko SV, Johnston SL (2009) Respiratory virus induction of alpha-, beta- and lambda-interferons in bronchial epithelial cells and peripheral blood mononuclear cells. Allergy 64(3):375-386. doi:10.1111/j.1398-9995.2008.01826.x

Kolumam GA, Thomas S, Thompson LJ, Sprent J, MuraliKrishna K (2005) Type I interferons act directly on CD8 T cells to allow clonal expansion and memory formation in response to viral infection. J Exp Med 202(5):637-650. doi:10.1084/jem.20050821

Kotenko SV, Gallagher G, Baurin VV, Lewis-AntesA SM, Shah NK, Langer JA, Sheikh F, Dickensheets H, Donnelly RP (2003) IFN- $\lambda$ s mediate antiviral protection through a 
distinct class II cytokine receptor complex. Nat Immunol 4 (1):69-77. doi:10.1038/ni875

Li M, Liu X, Zhou Y, Su SB (2009) Interferon- $\lambda$ s: the modulators of antivirus, antitumor, and immune responses. J Leukoc Biol 86(1):23-32. doi:10.1189/jlb.1208761

Liu YJ (2005) IPC: professional type 1 interferonproducing cells and plasmacytoid dendritic cell precursors. Annu Rev Immunol 23:275-306. doi:10.1146/ annurev.immunol.23.021704.115633

Mbawuike IN, Wyde PR, Anderson PM (1990) Enhancement of the protective efficacy of inactivated influenza A virus vaccine in aged mice by IL-2 liposomes. Vaccine 8 (4):347-352. doi:10.1016/0264-410X(90)90093-2

Mbawuike IN, Lange AR, Couch RB (1993) Diminished influenza A virus-specific MHC class I-restricted cytotoxic $\mathrm{T}$ lymphocyte activity among elderly persons. Viral Immunol 6(1):55-64

Mordstein M, Neugebauer E, Ditt V, Jessen B, Rieger T, Falcone V, Sorgeloos F, Ehl S, Mayer D, Kochs G, Schwemmle M, Günther S, Drosten C, Michiels T, Staeheli P (2010) Lambda interferon renders epithelial cells of the respiratory and gastrointestinal tracts resistant to viral infections. J Virol 84(11):5670-5677. doi:10.1128/ JVI.00272-10

Onoguchi K, Yoneyama M, Takemura A, Akira S, Taniguchi T, Namiki H, Fujita T (2007) Viral infections activate types I and III interferon genes through a common mechanism. J Biol Chem 282(10):7576-7581. doi:10.1074/jbc. M608618200

O'Shea JJ, Visconti R (2000) Type 1 IFNs and regulation of TH1 responses: enigmas both resolved and emerge. Nat Immunol 1(1):17-19. doi:10.1038/76872

Panda A, Qian F, Mohanty S, van Duin D, Newman FK, Zhang L, Chen S, Towle V, Belshe RB, Fikrig E, Allore HG, Montgomery RR, Shaw AC (2010) Age-associated decrease in TLR function in primary human dendritic cells predicts influenza vaccine response. J Immunol 184 (5):2518-2527. doi:10.4049/jimmunol.0901022

Pawelec G, Solana R, Remarque E, Mariani E (1998) Impact of aging on innate immunity. J Leukoc Biol 64(6):703-712

Powers DC (1994) Effect of age on serum immunoglobulin G subclass antibody responses to inactivated influenza virus vaccine. J Med Virol 43(1):57-61

Powers DC, Belshe RB (1993) Effect of age on cytotoxic T lymphocyte memory as well as serum and local antibody responses elicited by inactivated influenza virus vaccine. $\mathrm{J}$ Infect Dis 167(3):584-592

Robek MD, Boyd BS, Chisari FV (2005) Lambda interferon inhibits hepatitis B and C virus replication. J Virol 79 (6):3851-3854. doi:10.1128/JVI.79.6.3851-3854.2005
Schildgen O (2009) The lack of protective immunity against RSV in the elderly. Epidemiol Infect 137(12):1687-1690. doi:10.1017/S0950268809990690

Seeds RE, Gordon S, Miller JL (2006) Receptors and ligands involved in viral induction of type I interferon production by plasmacytoid dendritic cells. Immunobiology 211(68):525-535. doi:10.1016/j.imbio.2006.05.024

Shodell M, Siegal FP (2002) Circulating, interferon-producing plasmacytoid dendritic cells decline during human ageing. Scand J Immunol 56(5):518-521. doi:10.1046/j.13653083.2002.01148.x

Solana R, Pawelec G, Tarazona R (2006) Aging and innate immunity. Immunity 24(5):491-494. doi:10.1016/j.immuni.2006.05.003

Sommereyns C, Paul S, Staeheli P, Michiels T (2008) IFNlambda (IFN-lambda) is expressed in a tissue-dependent fashion and primarily acts on epithelial cells in vivo. PLoS Pathog 4(3):e1000017. doi:10.1371/journal.ppat.1000017

Stetson DB, Medzhitov R (2006) Type I interferons in host defense. Immunity 25(3):373-381. doi:10.1016/j. immuni.2006.08.007

Stout-Delgado HW, Yang X, Walker WE, Tesar BM, Goldstein DR (2008) Aging impairs IFN regulatory factor 7 upregulation in plasmacytoid dendritic cells during TLR9 activation. J Immunol 181(10):6747-6756

Takeuchi O, Hemmi H, Akira S (2004) Interferon response induced by Toll-like receptor signaling. J Endotoxin Res 10(4):252-256. doi:10.1177/09680519040100040901

Tiwari RK, Wong GY, Liu J, Miller D, Osborne MP (1991) Augmentation of cytotoxicity using combinations of interferons (types I and II), tumor necrosis factor-alpha, and tamoxifen in MCF-7 cells. Cancer Lett 61(1):45-52. doi:10.1016/0304-3835(91)90075-S

Treanor J, Falsey A (1999) Respiratory viral infections in the elderly. Antiviral Res 44(2):79-102. doi:10.1016/S01663542(99)00062-5

Watarai $\mathrm{H}$, Sekine $\mathrm{E}$, Inoue $\mathrm{S}$, Nakagawa $\mathrm{R}$, Kaisho $\mathrm{T}$, Taniguchi M (2008) PDC-TREM, a plasmacytoid dendritic cell-specific receptor, is responsible for augmented production of type I interferon. Proc Natl Acad Sci U S A 105(8):2993-2998. doi:10.1073/pnas.0710351105

Witte K, Gruetz G, Volk HD, Looman AC, Asadullah K, Sterry W, Sabat R, Wolk K (2009) Despite IFN-lambda receptor expression, blood immune cells, but not keratinocytes or melanocytes, have an impaired response to type III interferons: implications for therapeutic applications of these cytokines. Genes Immun 10(8):702-714. doi:10.1038/gene.2009.72

Yang OO, Lin H, Dagarag M, Ng HL, Effros RB, Uittenbogaart $\mathrm{CH}$ (2005) Decreased perforin and granzyme B expression in senescent HIV-1-specific cytotoxic T lymphocytes. Virology 332(1):16-19. doi:10.1016/j.virol.2004.11.028 